\title{
LA OCUPACIÓN DEL ALERO DE CERRO CASTILLO: UN SITIO RESIDENCIAL EN UN MIRADOR PANORÁMICO.
}

D. LEGOUPIL*

\section{RESUMEN}

El fondo de la antigua cuenca glaciar del lago Toro, localizada en la entrada del Parque Nacional Torres del Paine, es un área rica en sitios arqueológicos erosionados, en particular en el borde de los ríos. Éstos testimonian una expansión demográfica importante en la zona, si bien ésta es poco conocida y está mal datada. Nuevas excavaciones de un sitio arqueológico descubierto en un alero rocoso en Cerro Castillo podían documentar el desarrollo de estos grupos a partir del V milenio AP. (San Román y Morello 2003). Con este fin, la misión arqueológica francesa realizó un segundo sondeo en el sitio durante el año 2006. Este sondeo permitió un estudio paleoambiental del sitio (Bertrán 2009, Solari 2009, ambos en este volumen). El estudio de más de 10.000 vestigios líticos (Langlais y Morello 2009, en este volumen), y la realización de un primer análisis arqueozoológico (San Román y Morello 2003; Prieto en Legoupil y Bertrán 2006).

El conjunto de estos datos confirma el fuerte potencial informativo de este alero que, al ofrecer un control visual del inmenso valle representa un paradero excepcional para cazadores. Los testimonios de actividades cinegéticas son escasos (pocas puntas de proyectil y ninguna bola), mas el sitio presenta las características de un campamento habitacional en el que ocurrieron importantes actividades domésticas; así lo atestiguan la producción de lascas (a menudo utilizadas) y la abundancia de raederas, raspadores y cuchillos, próximas a áreas de combustión.

A pesar de las perturbaciones postdeposicionales típicas en tales contextos, y de la riqueza del palimpsesto de ocupaciones registradas, es posible distinguir grandes etapas de ocupación humana en el sitio. El Hombre se instaló hace 4500 años, en los primeros momentos de formación de la capa sedimentaria IV, un período húmedo que favoreció el desarrollo de una cubierta vegetal que también es registrada a continuación en la capa III antes de desaparecer en la etapa final del depósito: la estepa actual. En un contexto caracterizado por una tecnología común, se observan diversas modificaciones técnicas (quizás de origen funcional): por ejemplo, el abandono del uso de la obsidiana en favor de la calcedonia, o el reemplazo de las raederas por lascas retocadas y raspadores como herramientas dominantes. La distribución de los restos, la presencia de fogones y el uso de bloques rocosos, estos últimos tal vez dispuestos intencionalmente, ponen de manifiesto el potencial paleoetnográfico del sitio. Cerro Castillo podría contribuir a aclarar las características de los modos de vida de los cazadores terrestres de la región y ofrecer una columna estratigráfica bien controlada para la segunda mitad del Holoceno.

PALABRAS CLAVES: Cazadores terrestres, Holoceno medio, paleoambiente. 


\title{
CERRO CASTILLO ROCKSHELTER OCCUPATION: A RESIDENTIAL SITE IN A PANORAMIC VIEWING-POINT.
}

\begin{abstract}
The end of the former Lago Toro glacier basin, at the entry of Torres del Paine park, is a rich area with eroded archaeological sites, especially at the edge of rivers. These bear witness to an important demographic expansion in the zone, which, however, little known and poorly dated. An archaeological site discovered in a rock shelter at Cerro Castillo (San Roman and Morello 2003) might document these groups, from the $5^{\circ}$ millenium AP. With this purpose, a second test pit was done in year 2006 by the archaeological French mission, allowing a palaeoenvironmental study of the site (Bertrán 2009, Solari 2009, both in this volume). More than 10.000 lithic materials were studied (Langlais and Morello 2009), and a first archaeozoological analysis was done (San Roman and Morello 2003; Prieto in Legoupil and Bertran 2006).

These studies confirm the strong informative potential of this rock shelter which, by offering visual control of the immense valley, represents an exceptional where about for hunters. Nevertheless evidence of hunting activities is scanty (few projectile points and no bolas), instead the site shows characteristics of a habitation camp, with important domestic activities that testify the production of stone flakes (often used) and the abundance of sidescrapers, end-scrapers and knives in the proximity of combustion areas.

In spite of postdepositional disturbances, common in such contexts, and of the rich palimpsest of occupations observed, it is possible to distinguish broad stages of human occupation in the site. Man installed himself 4500 years ago, in the first moments of sedimentary unit IV, a humid period that favored the development of woodland cover, which is also recorded in unit III before disappearing in the final stage; where the current steppe predominates.

Within a common technological environment, different technical modifications are noted (probably of functional origin): for example, abandon sent of the use of obsidian in favour of chalcedony, and the replacement of side-scrapers for retouched flakes anwd end-scrapers, as dominant tools. The distribution of remains, the presence of fireplaces and the use of blocks, the latter possibly arranged intentionally, highlight the palaeoethnographic potential of this site. It might clarify the lifeways of terrestrial hunters in the region and offer a well controlled stratigraphic column coverig the second half of the Holocene.
\end{abstract}

KEYWORDS: Land hunters, middle Holocene, palaeoenvironment.

\section{INTRODUCCIÓN}

Después de veinticinco años de estudio de los comportamientos y modelos de adaptación de los grupos marítimos de Patagonia, el carácter tecno-económico mixto del sitio de Ponsonby nos llevó recientemente a interesarnos en los cazadores terrestres y sus sistemas técnicos. Ya se habían sugerido relaciones ocasionales entre estos dos grupos, por ejemplo entre Ponsonby y los cazadores de la Cueva Fell (Schidlowsky 2001). Un problema latente en estas comparaciones suscitaba, nuestro interés: el origen de las grandes puntas bifaciales foliáceas de Ponsonby.
El descubrimiento de puntas de morfología bastante similares, aunque generalmente más pequeñas, en la región de Puerto Montt (Gaete et al. 2004), Chiloé (Rivas et al. 1999), y en las islas Guaitecas (Porter 1993) sugirió la hipótesis de la difusión de rasgos culturales desde poblaciones del norte de los archipiélagos. Inicialmente, por tanto buscamos rastros de esta tradición técnica en el dédalo de los archipiélagos occidentales que separa las dos regiones. Sin embargo el único sitio de más de 4000 años descubierto en este medio difícil y de muy baja visibilidad arqueológica, una sepultura en cueva de la isla Madre de Dios, carecía de material cultural (Legoupil y Sellier 2005). La antigüedad de 
otros sitios registrados en Puerto Edén (Curry 1991, San Román com. pers.) y en la isla Stuven (Legoupil et al. 2005), apenas excede los primeros siglos de nuestra era y, adicionalmente, ésos se caracterizan por una gran escasez de material lítico incluidas la ausencia de puntas.

Entonces encaramos la posibilidad de una innovación vinculada a un mestizaje técnico entre cazadores terrestres y marítimos, cuyos rastros eran visibles en Ponsonby. Para comprobar esta hipótesis, sin embargo, era necesario descubrir un sitio terrestre contemporáneo a Ponsonby, no muy distante del territorio marítimo, y en el cual fueron observables las técnicas de trabajo bifacial. Más allá de este planteamiento anecdótico, el sitio debía permitir un enfoque global (tecnológico y, también económico y social), conforme a la tradición inductiva de la escuela francesa, fundada en la observación de hechos para formar sistemas y, a veces, modelos.

Los vestigios arqueológicos son muy ricos en el territorio terrestre de Patagonia; sin embargo los sitios con estratigrafía, bien fechados, son muy raros. Por eso elegimos el sitio de Cerro Castillo, en el que un equipo del Instituto de la Patagonia había descubierto a principios del 2000 ocupaciones humanas y evidencias de trabajo bifacial, asociadas con una fecha de $4.580 \pm 80$ AP (San Román y Morello 2003). Esta fecha corresponde cronológicamente a la fase cultural de grandes puntas bifaciales, que ha sido datada entre 4.200 a 4.600 años AP en la capa $\mathrm{B}$ de Ponsonby. La pequeña excavación realizada entonces fue reabierta y expandida, por un equipo franco-chileno en 2006. En total, sumadas las dos campañas, se recolectaron 10.650 piezas líticas, así como 1.663 restos óseos, la mayor parte de ellos concentrados en la mitad superior de la estratigrafía. Estos vestigios permitieron el análisis de los comportamientos técnicos de los ocupantes del sitio (Langlais y Morello 2009). El estudio geomorfológico efectuado por P. Bertrán (2009, en este volumen) permitió observar las condiciones de formación y de conservación de las capas naturales y de los suelos arqueológicos. El tamizado fino (malla de $2 \mathrm{~mm}$ ) de una columna de muestreo, permitió analizar la distribución de microrestos líticos, sobre un cuarto de metro cuadrado (ibid). Por fin se efectuó un análisis antracólogico para reconstruir la evolución de la vegetación durante la segunda mitad del Holoceno (M. E. Solari 2009, en este volumen).

\section{UNA REGION RICA PERO POCO ESTUDIADA}

La localidad de Cerro Castillo está ubicada en el fondo de la antigua cuenca glaciar del lago Toro y constituye la confluencia de vías de tránsito que dan acceso al macizo del Paine (al noreste), a las grandes planicies argentinas (al este) y al seno Última Esperanza, éste último ubicado a unos sesenta kilómetros al sur. Aún cuando el alero rocoso Cerro Castillo 1 es el primer sitio excavado en esta zona, su riqueza arqueológica fue señalada hace tiempo. Ya en los años de 1950, J. Emperaire y A. Laming escribían ${ }^{1}$ : La cuenca de Cerro Castillo parece un gran centro de asentamiento de una población bastante uniforme caracterizada por su industria sobre lascas de esquisto ${ }^{2}$. Se encuentra esta industria hasta la frontera, la Laguna Amarga, Cerro Payne, la Laguna Figueroa. El nivel básico parece ser el borde de la terraza del Río de Las Chinas pero los yacimientos remontan a lo largo de las cuestas, siguiendo las orillas de los ríos. Se puede decir que prácticamente por todas partes donde hay un lugar denudado, hay industria... ${ }^{3}$ (Emperaire et Laming, nd, p. 20). Alrededor de 500 piezas recogidas por estos autores en Cancha Carrera y en las orillas del Río Cóndor, hoy depositadas en el museo del Quai Branly en Paris, fueron el objeto de un inventario tipológico preliminar que muestra una representación de artefactos clásica: raspadores, raederas y cuchillos (Guidon y Laboudie n. d.). El trabajo bifacial es evidenciado por algunos bifaces y tres puntas pedunculadas (Fig. 1, a la izquierda), de tipo Bird IV (Bird 1993). Entre éstas, una punta sin pedúnculo (Fig. 1, a la derecha), evoca las puntas triangulares del tipo Bird III, así como también las puntas de Ponsonby, a las que se acerca por su gran

1 Los archivos Emperaire fueron repertoriados hace poco en el cuadro de un programo de cooperación franco-chileno (ECOS-CONICYT C04H01).

2 Probablemente lo que nombramos ahora como lutita.

3 Traducción de la autora. Original: "Le bassin de Cerro Castillo semble un grand centre de peuplement d'une population assez uniforme caractérisée par son industrie sur éclats de schiste. On retrouve cette industrie jusqu'à la frontière, la laguna Amarga, le cerro Payne, la laguna Figueroa. Le niveau de base semble être le bord de la terrasse du Rio de las Chinas mais les gisements remontent le long des pentes en suivant les rives des rios. On peut dire que pratiquement partout où il y a un endroit dénudé, il y a industrie ..." (Emperaire et Laming, nd, p. 20). 


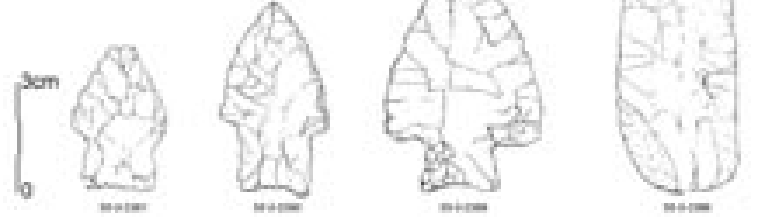

Fig. 1. Las puntas de Río Cóndor (Guidon y Laboudie, n. d.).

dimensión (105mm) y por la morfología de su base. Sin embargo, la característica más notable de éstas, el denticulado, está ausente.

En los años 1970 F. Bate descubrió un sitio de cazadores de guanacos, sobre una duna eólica ubicada en el margen este del lago Toro a una decena de kilómetros de Cerro Castillo. Es uno de los pocos sitios con estratigrafía observable en la región. Fue datado recientemente por el Instituto de la Patagonia en $3.615 \pm 40$ AP (Ua-21037), hoy está en curso de destrucción muy avanzada. Más recientemente, M. San Román y F. Morello (op. cit.) señalaron también la existencia de una treintena de sitios al aire libre, la mayor parte asignados al período Bird IV, cerca del Río Don Guillermo. Finalmente vestigios de piedra tallada han sido informados regularmente por aficionados, indicando la presencia de sitios de superficie erosionados próximos al pueblo de Cerro Castillo. Algunas de estas piezas están depositadas en un pequeño museo local. Sin duda la abundancia de estos hallazgos es un índice de la importan-

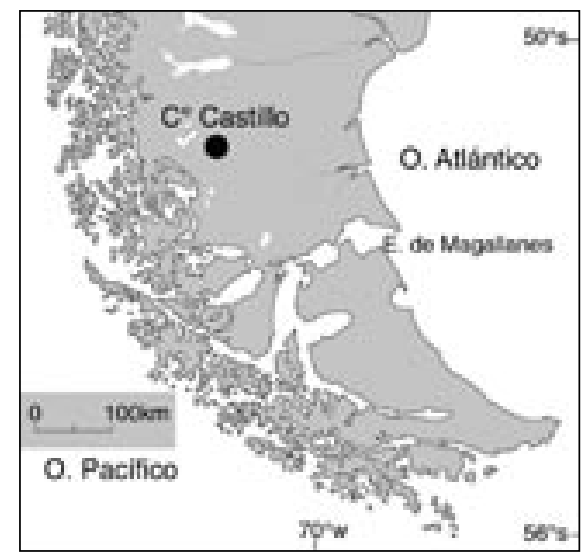

Fig. 2a. Ubicacion regional de el alero Cerro Castillo. cia de los asentamientos humanos en la región. Desgraciadamente la mayoría de los vestigios proceden de sitios erosionados $y$, por tanto, son difíciles de fechar. En realidad, los mejores controles cronológicos para la región provienen de sitios en cuevas o aleros rocosos, menos expuestos a la erosión que los lados del valle y los bordes de ríos o lagos. De ahí el interés del alero que sobresale al pueblo de Cerro Castillo. El objetivo inicial de San Román y Morello (2003) consistía en buscar rastros de ocupaciones paleoindias comparables a las de la zona de Cerro Benítez: Cueva Lago Sofía 1 (Prieto et al. 1991) y Cueva del Medio (Nami 1987). De hecho, el descubrimiento de un osteodermo de milodón indica la existencia de una capa finipleistocénica o de principios del Holoceno, a $1,80 \mathrm{~m}$ de profundidad. Pero este único vestigio no estaba asociado a huellas de actividad humana, siendo el sondeo obviamente muy reducido. Por el contrario las capas superiores, correspondientes a la segunda mitad del Holoceno, resultaban muy ricas.

\section{UN PARADERO ESTRATÉGICO}

El alero Cerro Castillo (Fig. 2a) ofrece una visión excepcional, de más $180^{\circ}$, sobre el valle glaciar del lago Toro. Es un lugar privilegiado que permite observar fácilmente todo movimiento animal o humano. El alero excavado es el más grande y profundo de una serie de anfractuosidades visibles en tres farellones escalonados (Fig. 2b), en alguno de los cuales se encontraron algunas piezas arqueológicas dispersas en superficie. Delante del gran alero, un

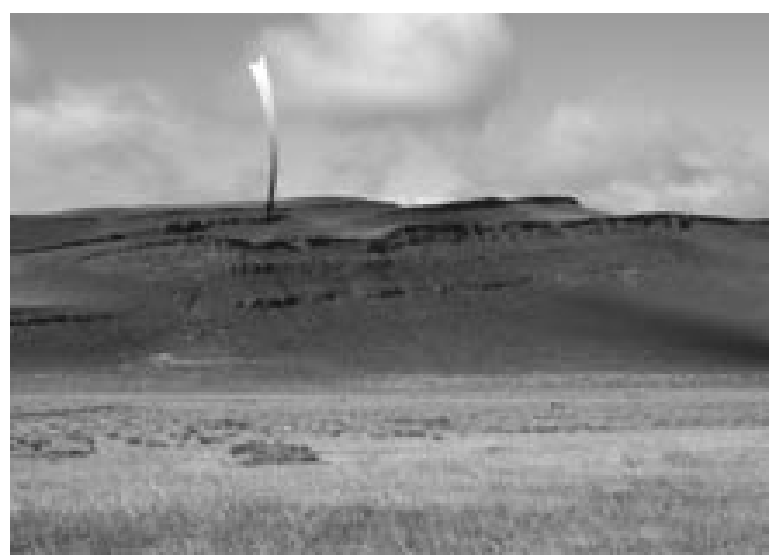

Fig. 2b. El alero de Cerro Castillo en las bardas rocosas. 


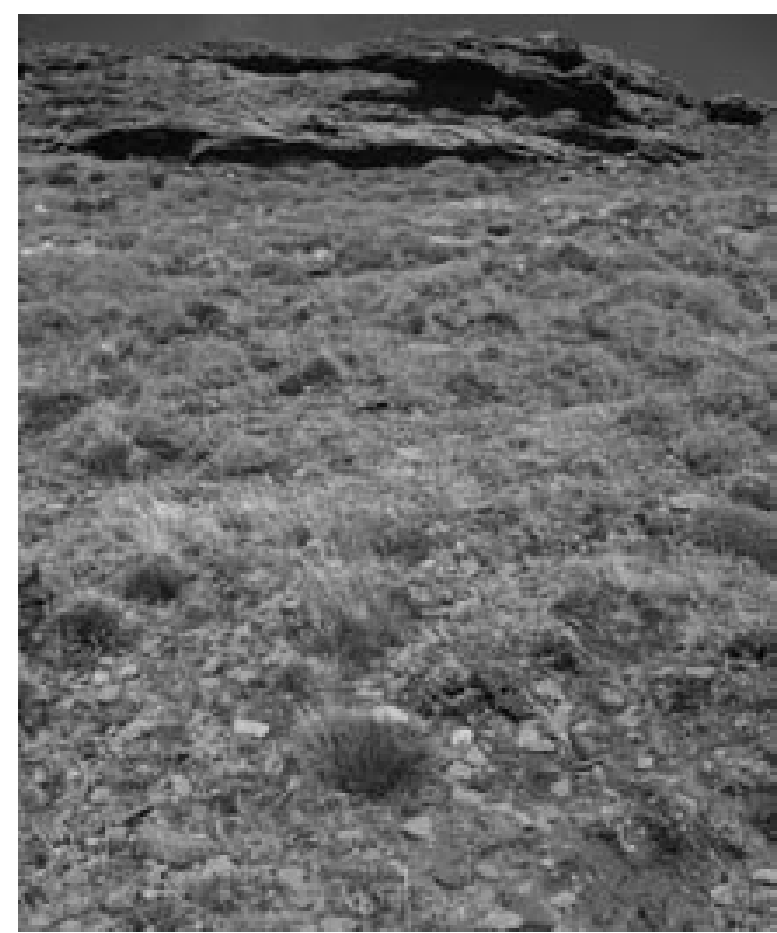

Fig. 3. Hay numerosos restos líticos y óseos en el cono de escombros delante del alero.

cono de escombros erosionado presenta numerosas piezas arqueológicas líticas y óseas redistribuidas por agentes eólicos y gravitacionales (Fig. 3). Es posible que aficionados locales ya hayan sesgado la muestra superficial. Ningún río pasa en las proximidades, pero al extremo septentrional de la barra rocosa del alero, una pequeña depresión húmeda y pantanosa, pudo proporcionar el indispensable acceso al agua durante una ocupación residencial, en particular en un período húmedo, o potencialmente, a través de la excavación de un pozo.

\section{LAS EXCAVACIONES}

Como resultado de las dos campañas de excavación en el sitio (D/E15 en la primera; F15 en la segunda) se obtuvo una trinchera de $2,50 \mathrm{~m}$ de longitud, $1 \mathrm{~m}$ de ancho y $2 \mathrm{~m}$ de profundidad máxima que cruza la parte central del alero (Fig. 4). Los niveles arqueológicos que correspondían a la segunda mitad del Holoceno (en el primer metro, de profundidad), quedaron excavados en casi toda la superficie abierta. El número de piezas arqueológicas se presentó especialmente elevado hacia la pared, en D/E15, donde se encontraba seguramente el núcleo de las ocupaciones. Por razones de acceso y seguridad hubo que hacer escalones, lo que limitó progresivamente la superficie excavada a las dos extremidades. De esta manera los niveles correspondientes a la primera mitad del Holoceno sólo fueron alcanzados en E15 y parcialmente en F15, mientras que la excavación del nivel más antiguo se limitó a un área inferior a un metro cuadrado. Las características de estas unidades depositacionales serán descritas a continuación.

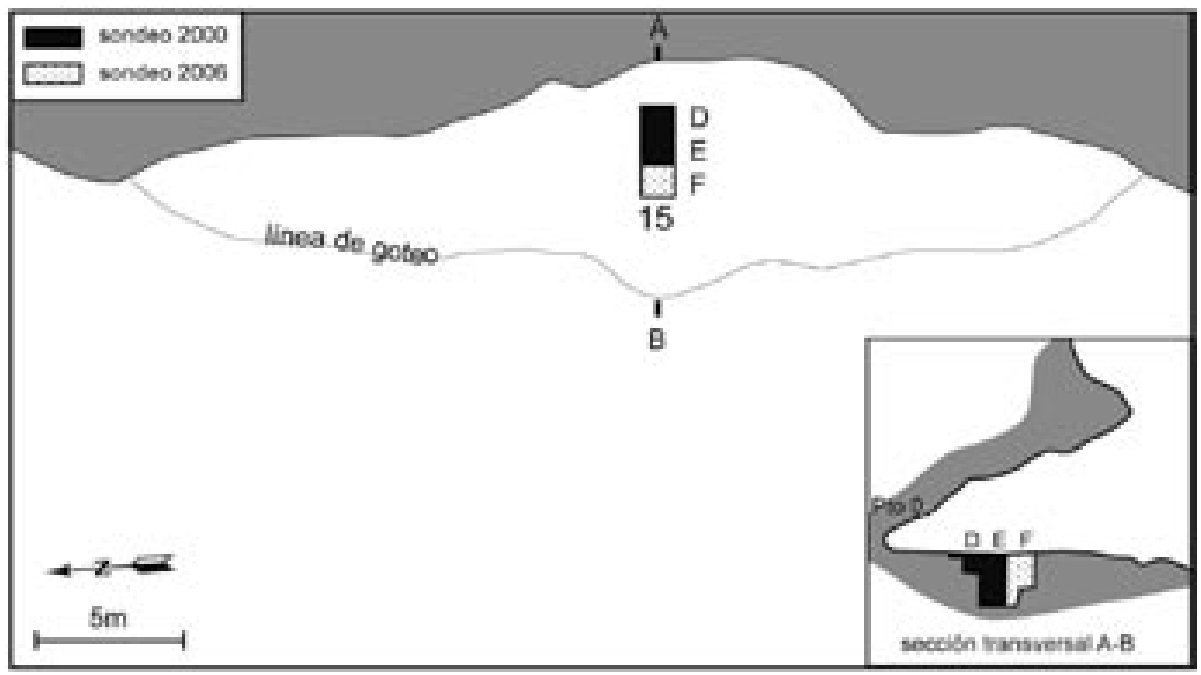

Fig. 4. Las excavaciones en el alero Cerro Castillo: plano y sección (modificado de San Román y Morello 2003). 


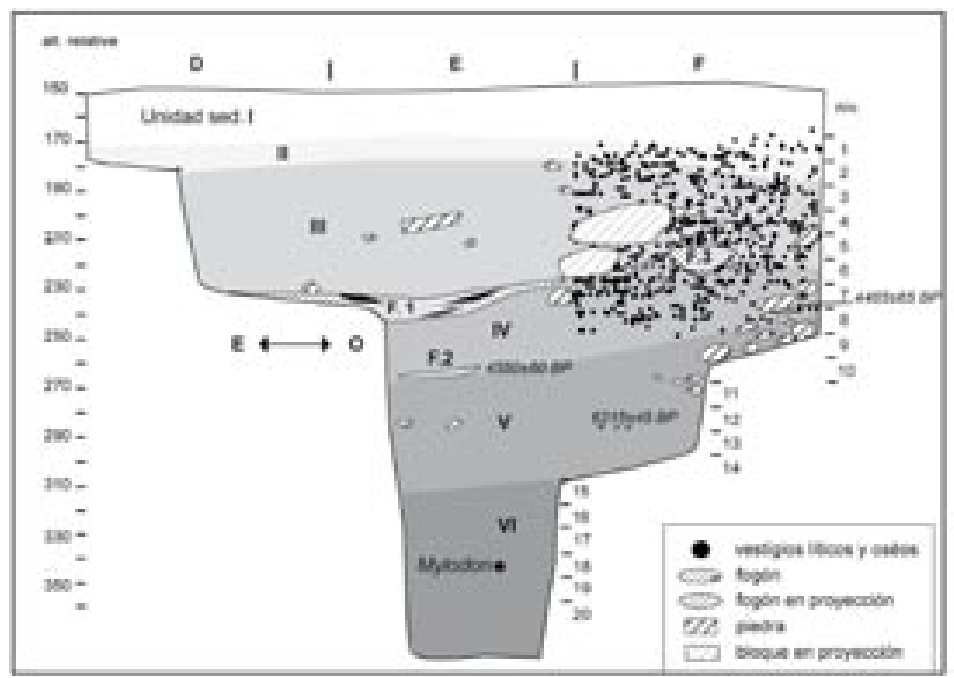

Fig. 5. Perfil sur de la excavación y proyección de los vestigios y estructuras (según Morello y San Román 2003, y Bertrán 2009).

\section{LA FORMACION DE LAS CAPAS SEDIMENTARIAS Y LA EVOLUCIÓN DEL PAISAJE}

La secuencia obtenida en las excavaciones cubre todo el Holoceno. Las modificaciones naturales del medio ambiente son evidenciadas por las variaciones pedológicas de las capas y la alteración de las piezas óseas (Bertrán 2009). Se definieron seis unidades sedimentarias (Fig. 5). En los niveles superiores, estas son relativamente horizontales, debido a un relleno progresivo de la sedimentación gravitacional; en cambio los sedimentos presentan una fuerte inclinación hacia el interior del alero, en las capas inferiores. La secuencia estratigráfica comienza casi a $2 \mathrm{mt}$ de profundidad: ésta representa el final del Pleistoceno o el inicio del Holoceno (Fig. 5, unidad sedimentaria VI) como parece indicarlo la naturaleza de los sedimentos y la presencia del hueso de milodón. Para obtener una precisión mayor será necesario realizar un fechado directo, especialmente porque el período de extinción de estos grandes perezosos está aún en discusión (Borrero 1997).

Las unidades sedimentarias inferiores (VI y V) están constituidas por coluviones detríticos arenosos, con escasa coloración orgánica, lo que refleja un ambiente con escasa vegetación. Según P. Bertrán (ibid.), la estepa habría durado más tiempo que en la región del Cerro Benítez por razones de exposición local. En estas capas, los restos arqueológicos eran raros y quizá intrusivos.

En la unidad V, a 1,45 m de profundidad, un pequeño lente de esquirlas líticas y óseas, en F15, fue datado en $6.215 \pm 45$ años AP. Según P. Bertrán, este fino depósito, parcialmente arenoso, podría ser resultado de un escurrimiento natural en una grieta, debidao a ciclos de hielo/deshielo y en consecuencia podría proceder de un nivel ligeramente superior.

La base de la unidad sedimentaria IV, que corresponde al principio de las ocupaciones arqueológicas, se caracteriza por el fogón 2 descubierto en E15 y datado en $4.580 \pm 80$ AP (Morello y San Román 2003). Se obtuvo otro fechado muy comparable (4.485 \pm 65 AP, Gif-12225) sobre carbones depositados bajo una piedra en F15, en una posición un poco más alta, pero continua al considerar la inclinación natural de la capa. Esta unidad está constituida por un paleosuelo alterado (descarbonatado), que sugiere un ambiente vegetal mucho más importante. Correspondería a un período más húmedo, también señalado en los datos palinológicos regionales (cf. Moreno et al. 2009, en Bertran 2009).

Por fin, las unidades III y II están constituidas por sedimentos coluviales entre los cuales se intercalan regueros de arenas y gravas, que sugieren un escurrimiento que potencialmente, desplazó localmente los vestigios arqueológicos. El sedimento, 


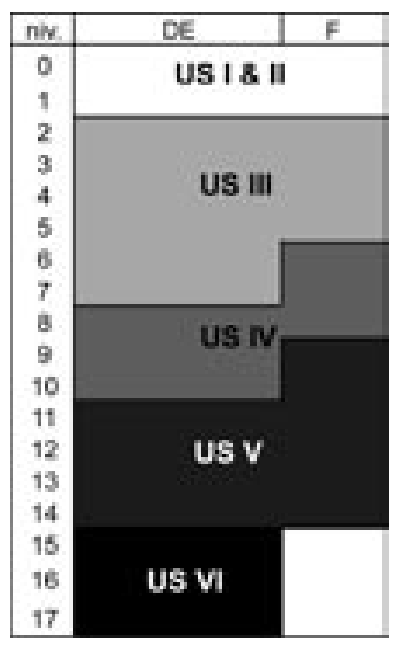

Fig. 6. Esquema de correspondencia de los niveles arqueológicos artificiales y las unidades sedimentarias (US).

menos descarbonatado, tiene un contenido orgánico intermedio. Estas unidades parecen representar un período más seco y una regresión progresiva del bosque que, ayudado por la colonización antrópica de los últimos siglos, llevó, a la estepa actual (ibid.). Debido seguramente a la acción del ganado moderno, la parte cubierta del alero está actualmente desprovista de vegetación, a excepción de algunos pequeños arbustos en el límite exterior del abrigo rocoso, bajo la línea de goteo. La estratigrafía termina con una gruesa capa de excrementos de ovejas (unidad I) depositada durante el siglo XX. En esta se puede observar algunos vestigios desplazados por el pisoteo animal, y al igual que sedimentos coluviales, asociados a la fuerte pendiente lateral de la superficie actual del alero.

\section{LA ESTRATIGRAFIA ARQUEOLOGICA}

Las excavaciones fueron efectuadas siguiendo niveles artificiales de $10 \mathrm{~cm}$ (numerados de 1 a 17), luego de traspasar la gruesa capa de excremento animal que constituye la unidad sedimentaria I. La capa arqueológica es más espesa en el fondo del alero (cuadrículas DE), que hacia el talud exterior (en F). En las zonas de transición de las unidades sedimentarias, producto de la pendiente observada, los niveles artificiales recortan los estratos identificados (Fig. 6). Por ello, los conjuntos arqueológicos se agruparon en función de su atribución sedimentaria para su análisis.

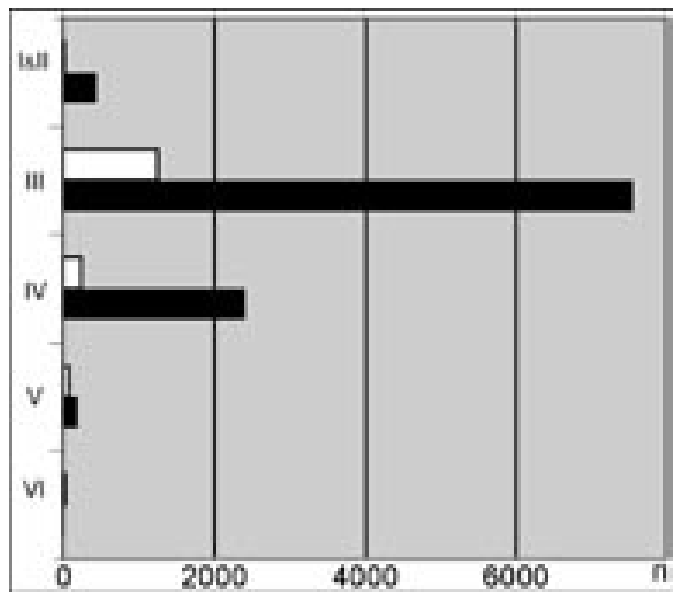

Fig. 7. Representación de los restos líticos y óseos en las unidades sedimentarias.

$\mathrm{Al}$ principio de la secuencia (unidades $\mathrm{V}$ y $\mathrm{VI}$ ), las restos arqueológicos fueron escasos (figura 7, tabla 1). Representan un $2 \%$ del material lítico (sobre todo esquirlas, más un percutor y un cuchillo), y un 7\% de las piezas óseas (astillas muy alteradas cuyo origen antrópico no se ha comprobado). Algunos vestigios formaban pequeños estratos, muy finos, en el nivel V. Éstos podrían ser resultado de infiltraciones y no constituir verdaderos niveles de ocupación.

$\mathrm{Al}$ contrario, en la parte superior de la estratigrafía, las dos primeras unidades sedimentarias estaban muy entremezcladas con pequeñas gravas y escombros. Éstas estaban depositados en posición casi horizontal debido al deslizamiento gravitacional del sedimento coluvial; a un aplanamiento probablemente acelerado por el pisoteo de las ovejas y a la desaparición de la vegetación, también vinculado al ganado. No se observó ninguna estructura, excepto un pequeño estrato rubificado en el perfil sur. Se

Tabla 1. Número de restos faunísticos y líticos ${ }^{4}$.

\begin{tabular}{|c|c|c|}
\hline U. sed. & fauna (NR) & líticos (n) \\
\hline I y II & 28 & 448 \\
\hline III & 1267 & 7568 \\
\hline IV & 245 & 2418 \\
\hline V & 86 & 196 \\
\hline VI & 40 & 20 \\
\hline total & 1666 & 10650 \\
\hline
\end{tabular}

4 Según las determinaciones de San Román y Morello 2003 (tabla 3) y A. Prieto ( Legoupil y Bertrán 2006) para la fauna; Langlais y Morello (en este volumen) para el material lítico. 
destacaron algunos vestigios, sobre todo líticos, entre los cuales mencionamos varias raederas y un pequeño raspador unguiforme en calcedonia. Los pocos restos óseos (1,4\% del total) estaban relativamente enteros, pero muy desgastados y agrietados por su exposición a las inclemencias del tiempo.

Entre estos dos extremos, la parte más importante de las ocupaciones arqueológicas se observa en las unidades medias III y IV, que contienen 94\% del material lítico y 91\% del material óseo. En estas unidades, que se extienden por casi $80 \mathrm{~cm}$ de espesor, la distribución de los vestigios es relativamente continua, probablemente debido a la escasa sedimentación entre ocupaciones humanas, a la importancia del escurrimiento, y a las bioturbaciones (raíces, animales fosoriales). Sin embargo, gracias a diferencias en los sedimentos, variaciones estadísticas en la representación de vestigios arqueológicos, y la presencia de algunos elementos estructurales (fogones $e$ instalaciones artificiales de bloques rocosos), es posible percibir distintas fases de ocupación.

\section{Las ocupaciones de la unidad IV}

Las primeras ocupaciones se detectaron en la unidad sedimentaria IV, entre los niveles 8-10 de las cuadrículas DE15, y 6-8 en F15. En F15 el espacio era limitado por la presencia de numerosos escombros depositados en forma paralela a la pendiente del talud (Fig. 8).

En el límite inferior de esta unidad se observó una fina capa endurecida por reprecipitación de carbonatos, en la que se registran algunos huesos muy deteriorados en una ganga de manganeso. Inmediatamente arriba, en E15, el fogón 2, datado por San Román y Morello (2003) en $4.580 \pm 80$ años AP, representa la primera ocupación. Esta estaría asociada al inicio de un periodo más húmedo con una vegetación arbórea más importante. Estos vestigios están asociados también con un pequeño lente de combustión rojo y negro, y algunas piedras quemadas que podrían representar un pequeño fogón anexo (véase Fig. 5, fogón 3 y Fig. 9), en la unidad F15.

Un fechado de $1.971 \pm 40$ años AP (Erl-10194), obtenido sobre esquirlas óseas bastante deterioradas del nivel 7 en F15, es poco creíble, especialmente si se considera su posición estratigráfica vinculada al

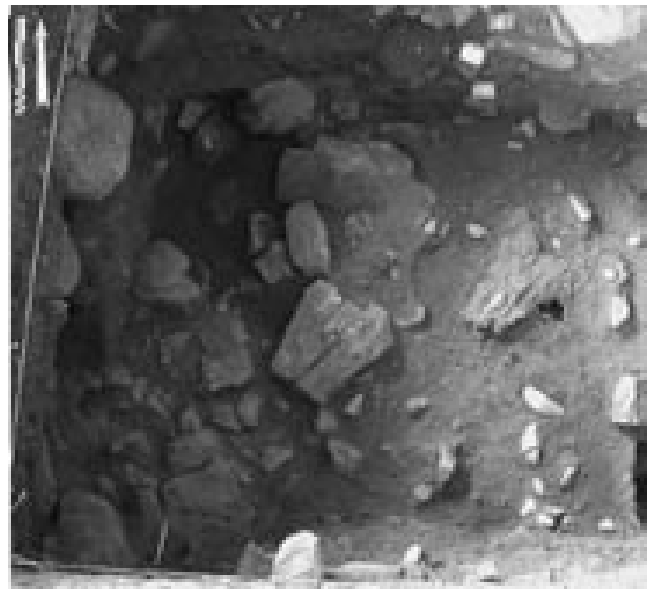

Fig. 8. Los escombros del nivel 7 en F15.

fogón 2 y la velocidad de sedimentación del alero. Las fases de ocupaciones se suceden sobre una treintena de centímetros de estratigrafía hasta el fogón 1 . Este fogón, muy grande y constituido de varios lentes de combustión, parece haber funcionado de manera intensiva durante el período de transición entre las unidades IV (en el cual forma una cubeta) y III. Representa probablemente el período de ocupación máxima del alero. En la unidad IV, la fauna es escasa, $15 \%$ del total de los vestigios, probablemente debido a la mala conservación de los huesos (Fig. 10). Ésta última fue observada por F. Martín en un primer estudio tafonómico (cf. Bertrán, este volumen) y confirmada gracias al análisis de un hueso del nivel 7 (F15) realizado por C. Chadefaux en el C2RMF (Centre de Recherche et de Restauration des Musées Français). El hueso, muy alterado, presentaba un

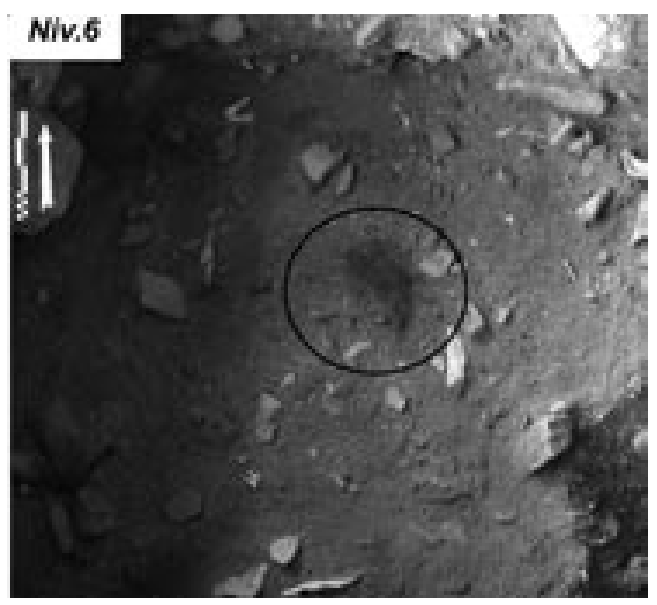

Fig. 9. El fogón 3 en F15. 


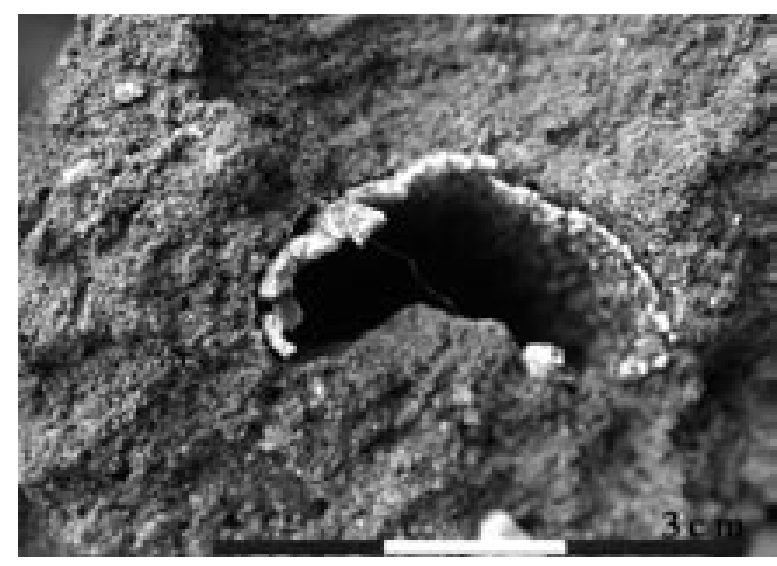

Fig. 10. Hueso de artiodáctilo alterado en la unidad sedimentaria IV: la pared del hueso, muy fina, está al límite de la destrucción.

elevado índice de cristalinidad $(0,38 \pm 0,01)$ lo que indica una fuerte desmineralización; también la presencia de microcanales rodeados de una fase más compacta, sugiere un ataque microbiano que "puede ponerse en relación con la presencia de humus procedente de la descomposición parcial de residuos vegetales (o animales) por micro-organismos"5 (Chadefaux 2006:44). Vinculamos estas observaciones con el paleosuelo mencionado por Bertrán y también con la probable acumulación de residuos de origen humano durante las ocupaciones pretéritas.

Los análisis faunísticos preliminares efectuados por San Román y Morello (2003) para DE15, y A. Prieto (en Legoupil y Bertrán, 2006) para

5 "peut être mise en relation avec la présence d'humus provenant de la décomposition partielle de déchets végétaux (ou animaux) par des micro-organismes" (Chadefaux 2006, p. 44)

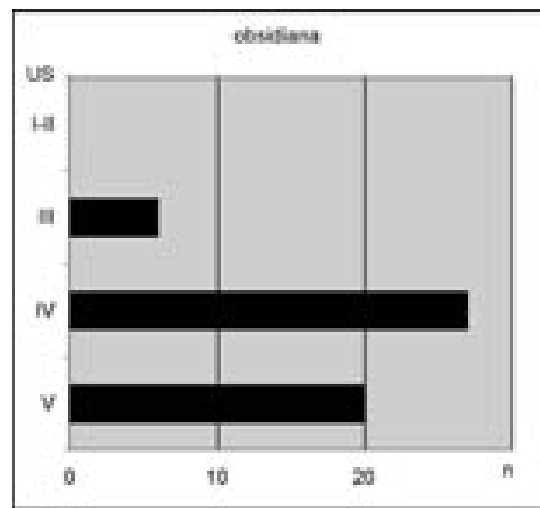

Fig. 11. Representación de los vestigios de obsidiana
F15, muestran la presencia exclusiva de mamíferos terrestres. Sólo se registraron guanacos, entre los cuales se identificó al menos en el nivel 6 (F15), un individuo joven, representado por 2 incisivos $y$ un fragmento de diáfisis. La presencia de guanaco juvenil pudo ser más importante en el sitio, pero su representación pudo verse desfavorecida por la conservación diferencial de sus restos. Los materiales líticos en estas primeras ocupaciones representan un 23\% del total (véase Tabl. 1). Como en todo el sitio, son sobre lutita (91\%), seguidos esencialmente por ríolitas y materiales silíceos (Langlais y Morello 2009, tabl. 1). Más sorprendente es la presencia de obsidiana, cuya presencia está restringida a las ocupaciones más antiguas (Fig. 11). Según el estudio de Langlais y Morello, una cincuentena de fragmentos y esquirlas de obsidiana gris, a veces negra, más raramente verde (como la de los archipiélagos, procede esencialmente del fondo de la unidad IV y de la parte superior de la unidad V. Su concentración en los niveles antiguos es confirmada por el tamizado fino de las esquirlas microlíticas efectuado por Bertrán (fig. 12). Por último las dos únicas puntas bifaciales descubiertas (en las excavaciones San Román y Morello) son también de obsidiana gris vetada y se encontraban, como el fogón 2 , al límite entre las dos unidades (en los niveles 10 en E15 y F15).

Entre las herramientas descubiertas en este conjunto dominan de manera clara las raederas que representan un $57 \%$ del total de la excavación y un $44 \%$ de los instrumentos de esta unidad (Tabl. 2 y Fig. 12). Estas raederas, simples o dobles, a veces convergentes, son a menudo grandes (hasta una decena de centímetros de longitud) y finas gracias a retoques escalariformes: recuerdan bastante las

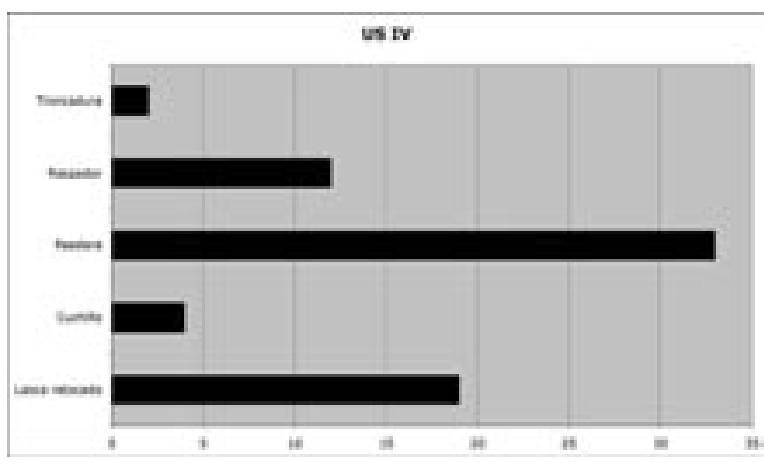

Fig. 12. Las principales herramientas enteras y fragmentos de herramientas de la unidad sedimentaria IV. 
Tabla 2. Las herramientas y fragmentos de herramientas (basado en el inventario realizado por M. Langlais).

\begin{tabular}{|c|c|c|c|c|c|c|c|c|c|c|c|c|c|c|}
\hline & $\begin{array}{l}0 \\
\mathbb{0} \\
0 \\
0 \\
0 \\
0 \\
0 \\
0 \\
0 \\
.\end{array}$ & $\begin{array}{l}\stackrel{0}{\vec{z}} \\
\text { ⿹ } \\
\text { J }\end{array}$ & 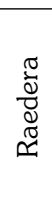 & 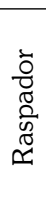 & 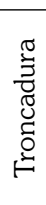 & 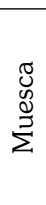 & 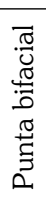 & 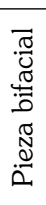 & ळు & 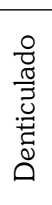 & 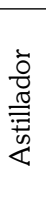 & $\frac{\stackrel{0}{\sigma}}{\xi}$ & 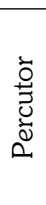 & $\begin{array}{l}\bar{\sigma} \\
0 \\
0\end{array}$ \\
\hline I-II & 3 & & & & & & & & & & & & & 3 \\
\hline III & 43 & 10 & 24 & 25 & 8 & 2 & & 5 & 5 & 3 & 1 & 1 & 1 & 128 \\
\hline IV & 19 & 4 & 33 & 12 & 2 & & 2 & & & 1 & & & 2 & 75 \\
\hline $\mathrm{V}$ & & & & 1 & & & & 1 & & & & & 1 & 3 \\
\hline Total & 65 & 14 & 57 & 38 & 10 & 2 & 2 & 6 & 5 & 4 & 1 & 1 & 4 & 209 \\
\hline
\end{tabular}

grandes raederas de la capa $\mathrm{B}$ de Ponsonby. Al igual que en este último sitio, casi todas las raederas del alero están hechas sobre lutita, material blando y poco agresivo. Estaban concentrados en la parte superior de la unidad IV (29 sobre 33 yacían en los niveles 6/7 de F15 y 8 de DE15). Representan una capa de ocupación bien definida, vinculada al gran fogón 1.

Otras herramientas, presentes en cantidades muy inferiores a las raederas, incluyen lascas retocadas lateralmente y los raspadores.

La unidad sedimentaria IV, por tanto, permite definir al menos dos fases de ocupación: una, vinculada al fogón 2 , que se caracterizada por la presencia de obsidiana en la cual están las únicas puntas proyectiles del sitio; otra, vinculada a la base del fogón 1, caracterizada por la abundancia de raederas, algunas de grandes dimensiones. Estas dos fases de ocupación fueron también distinguidas por P. Bertrán, en el análisis de los micro-vestigios (minerales, vegetales y animales) en F15. (Capas A4 y A3 Bertrán 2009, fig. 12).

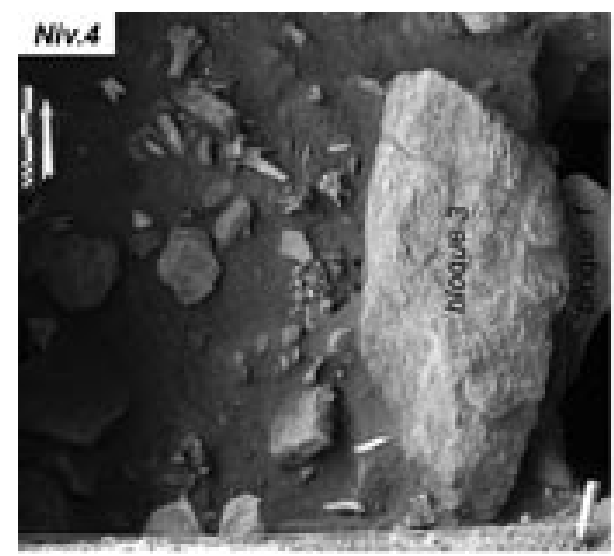

Las ocupaciones de la unidad sedimentaria III

La unidad sedimentaria III presenta un espesor de aproximadamente $50 \mathrm{~cm}$. Corresponde a un período menos húmedo que llevaría aparejado una regresión progresiva de la cubierta boscosa. En estos niveles se registró la mayor densidad de vestigios (véase Tabl. 1), tanto líticos (7.568 restos, 71\%) como óseos (1.267 restos, 76\%). El fogón 1, cuya base se vinculaba al nivel con grandes raederas, se inserta en cubeta en la unidad IV, lo que sugiere que siguió funcionando al depositarse en los primeros niveles de la unidad III. Según P. Bertrán, el fogón 1, es en realidad un horizonte orgánico oscuro limitado a $\mathrm{DE} 15$, en el que se observan varios lentes de combustión.

En esta unidad sedimentaria tres bloques amontonados, que pesan varios kilogramos o decenas de kilogramos cada uno, separan el espacio entre F15 y E15 (Fig. 5 y 13); estos bloques proceden muy ciertamente del techo, y aunque convendría re-examinar atentamente sus cicatrices, parece que

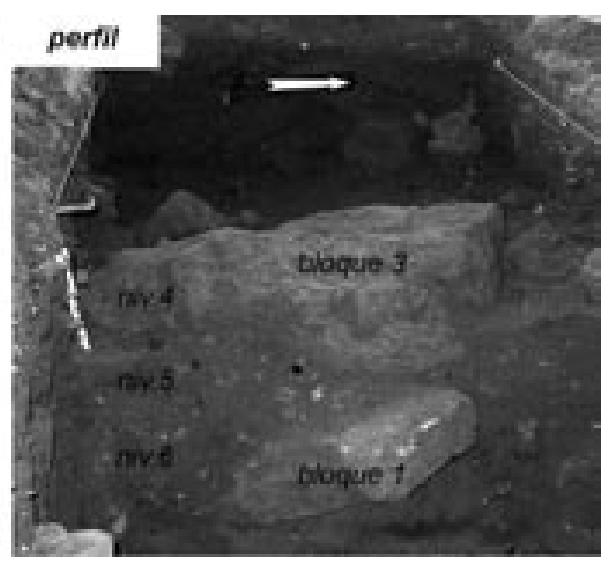

Fig. 13. Los bloque rocosos y la estructuración del espacio en la unidad III, en F15. 


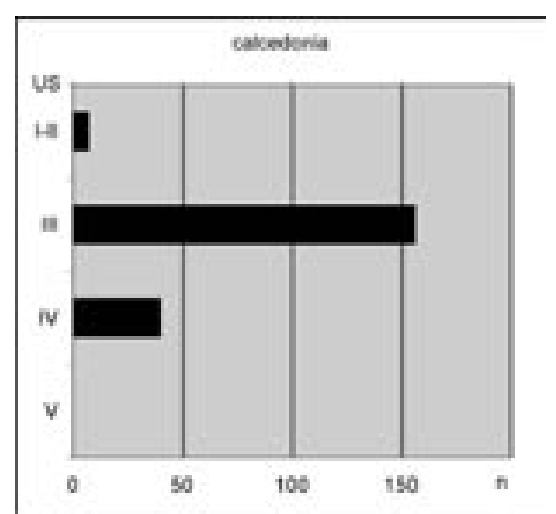

Fig. 14. Representación de los vestigios de calcedonia.

fueron alineados y superpuestos intencionalmente. Los dos más pequeños, probablemente fueron instalados al principio de la utilización con el objetivo de establecer un fogón, para hacer fuego: habrían protegido el lugar de las inclemencias del tiempo. Por el contrario, la pequeña estructura de combustión $n^{\circ} 3$, del otro lado de los bloques, quedaría expuesta al viento. La instalación del bloque más grande (Fig. 13, bloque 3), es posterior. Certifica una voluntad de mantenimiento, incluso de refuerzo, de la estructura. Este esbozo de muro formaba quizá parte de una estructura más compleja, reforzada por elementos perecibles o por la presencia de arbustos. Sería interesante comprobar si continúa en F14 y/o F16.

Los ocupantes de la unidad III utilizaron materias primas líticas muy comparables a las de la unidad IV, pero con dos diferencias notables: la obsidiana ya casi no se usa (se detectó solo una docena de vestigios) y, entre los materiales raros, se observa la presencia de numerosas piezas de calcedonia (Fig. 14).

Las herramientas más representadas son las lascas retocadas, seguidas por los raspadores. Las raederas, tan abundantes en la U.S. inferior, no están sino en tercera posición. Los raspadores (dos unguiformes) son manufacturados mayoritariamente en materiales líticos duros (calcedonia, sílex, jaspe). Esta elección prevalece en todas las capas. En total, sólo 12 raspadores solamente (de 38) están hechos de lutita, que es más blanda; entre ellos, 3 tenían también la función de raedera lo que implica que este uso determinó la elección de la materia prima. Parece pues, que los materiales más duros y mordaces fueron preferidos sistemáticamente para la

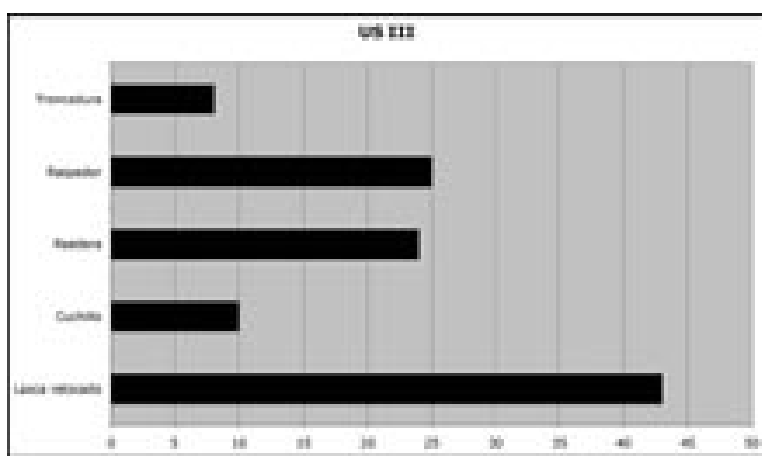

Fig. 15. Las principales herramientas de la unidad III.

confección de raspadores, mientras que la lutita, más blanda, fue privilegiada para la confección de las raederas. Es de gran interés notar que en el sitio de Ponsomby se observaron elecciones idénticas para la talla de lascas y la formalización de puntas o raederas (Pigeot 2003:126).

Según el estudio de Langlais y Morello (2009), aparecen en esta unidad sedimentaria numerosas piezas del tipo split o pièces esquillées (17 sobre un total de 20 en el yacimiento), éstas tal vez pudieron, quizás, servir de herramientas intermedias o funcionar como núcleos bipolares por percusión sobre yunque. Aunque raro, el trabajo bifacial está representado por 3 núcleos centrípetos bifaciales y dos piezas, no se hallaron puntas. En la parte superficial de esta unidad (E15 nivel 3), un fragmento de arenisca acanalado fue interpretado por San Román y Morello (2003) como alisador de astiles. El arco sólo apareció en Patagonia durante los primeros siglos de nuestra era (Prieto 1994), lo que permitiría suponer que la parte superior de esta unidad correspondería, como máximo, a esta época. Un fechado del sector medio de la unidad III (F15, nivel 4) está en curso.

Finalmente, varios huesos largos de guanaco con "corte perimetral" son registrados por San Román y Morello (ibid.); otro apareció en el nivel 4, en F15 (Fig. 13 arriba, a la izquierda). Estas piezas consideradas como "machacadores" por Hadjuk y Lezcano (2005), serían las únicas herramientas en hueso descubiertas en el alero, pero no son adecuados como marcadores cronológicos. Entre los restos faunísticos de la unidad III, algunas piezas presentan fisuras o exfoliaciones probablemente resultado de su exposición a las inclemencias del 
tiempo. En este nivel sin embargo los restos están, en su mayoría, poco alterados. El análisis de un hueso del nivel 5 (F15) confirmaba esta observación visual: presentaba un índice de cristalinidad bajo, y una elevada tasa de carbonatación secundaria de 0,39 \pm 0,03 (cf. Chadefaux op cit.), seguramente vinculada a la débil descarbonatación del sedimento. Ésta se debería a una pluviometría más moderada que en la unidad IV. Como en la unidad anterior, el hueso presenta, índices de ataque bacteriano (ibid.) que dan prueba de la presencia de humus, seguramente favorecido por las actividades humanas. La fauna, como en la U.S. inferior, es dominada casi exclusivamente por mamíferos terrestres. Entre ellos, sólo se identificaron huesos de guanaco. Se trata principalmente de adultos y también de algunos jóvenes, éstos representados por una media docena de huesos en F15. El origen antrópico de algunos pocos huesos de cánidos y aves, y de un hueso tarsiano de puma, requiere confirmación, visto su carácter excepcional.

\section{CONCLUSION}

Los sondeos realizados muestran el gran potencial arqueológico del sitio de Cerro Castillo 1 , especialmente en un período contemporáneo con la ocupación de la capa $\mathrm{B}$ de Ponsonby. Esta época, que comienza en el $5^{\circ}$ milenio AP, parece indicar una importante expansión demográfica que coincide con la instalación de una cubierta forestal, en la región(ello a pesar de que la biomasa de grandes mamíferos suele ser más baja en las zonas boscosas que en la estepa). Fechados complementarios deberían fijar la duración de estas ocupaciones. La industria lítica, muy rica, testimonia una tecnología que, segúnr Langlais y Morello, se expresa a lo largo de la secuencia: utilización preferencial de la lutita, desbaste uni y bifacial de núcleos (tipos discoidales y Levallois), talla sobre yunque, talla laminar ocasional (ya reconocida por Emperaire a partir de sus recolecciones superficiales), etc.

Como lo indica la importancia de la producción de lascas (a menudo utilizadas), y la abundancia de algunas herramientas (cuchillos, raspadores, raederas) destinadas al trabajo de pieles, de la madera, y/o a actividades de carnicería, la zona excavada corresponde claramente a actividades domésticas desarrolladas en la proximidad de los fogones.
La situación del alero es excepcional: un paradero que permite controlar visualmente un inmenso valle que fue tal vez una importante vía de comunicación entre el Cerro Benítez, los grandes llanos argentinos y el macizo del Paine. A pesar de esta situación privilegiada, los vestigios de actividades cinegéticas por el momento son raros. Las bolas, tan típicas de los cazadores de guanacos están ausentes. Las puntas proyectil son escasas (solamente dos pequeñas, en obsidiana, ambas en la base de la secuencia): un único hallazgo de un alisador indica, quizás, la fabricación de flechas en las capas superiores.

Los habitantes de Cerro Castillo no se interesaron en fabricar grandes puntas bifaciales, por lo menos en la zona excavada. Por ello la comparación con Ponsonby, nuestro inicial objetivo, no resulta por ahora posible. Es claro, sin embargo, que disponían del material preferido para la confección de puntas, un tipo de lutita en abundancia. Es claro también que habrían sido técnicamente capaces de fabricarlas, practicando tanto la talla bifacial, como la percusión dura, la percusión blanda, e incluso la presión (sobre obsidiana). De hecho es evidente que manejaron estas técnicas distramente, para la fabricación de raederas grandes, finas, con retoque escaleriforme, uni y a veces bifaciales, algunas muy similares a unas raederas de Ponsonby, así de típicas que fueron consideradas como "fuertes marcadores de identidad" (Pigeot 2003). Estas raederas están también representadas en el componente Antiguo de Lancha Packewaia y en la Cueva Fell (Schidlowsky 2001). Más allá de la presencia/ausencia de las grandes puntas, estas raederas son testimonio de una permeabilidad y relación sorprendentes entre las culturas con grandes puntas y los cazadores terrestres del $V^{\circ}$ milenio AP. Pero la fabricación de grandes puntas bifaciales lanceoladas por el momento, sigue siendo, una particularidad cultural muy significativa de los grupos costeros, tal vez producto de aspectos funcionales, o indicando una especificidad geográfica y/o cronológica marcada.

La escasez de armas y el gran número de herramientas significa que Cerro Castillo debió ser un sitio habitacional y no un simple paradero de caza, como ya lo habían notado San Román y Morello (2003). Sin embargo, considerando la poca superficie

6 "forts marqueurs identitaires" Pigeot 2003:148. 
excavada en relación al tamaño de este yacimiento, no se puede excluir que las armas fueron almacenadas y/o fabricadas en otras zonas, especializadas, en el alero o al exterior. La ausencia de puntas y bolas en el gran cono de escombros ubicado delante del alero no es significativa, siendo estos vestigios los más deseados por los coleccionistas y saqueadores. A pesar de haber perturbaciones postdeposicionales importantes, habituales en aleros, el resultado de los estudios geomorfológicos y antracológicos, la presencia de estructuras, y la distribución estratigráfica especializada de los vestigios (las raederas, las piezas tipo split, los raspadores unguiformes, los restos de obsidiana o calcedonia) ponen de manifiesto que un estudio paleoetnográfico es posible en este sitio. Dicho estudio podría aclarar los modos de vida de los cazadores terrestres de la región y ofrecer una columna estratigráfica bien controlado tanto del período clave del $\mathrm{V}^{\circ}$ milenio AP, como de los períodos posteriores.

\section{AGRADECIMIENTOS}

Los trabajos realizados en el año 2006 fueron financiados para la DGCID (Direction de la Coopération Internationale et du Développement) del Ministerio de Relaciones Exteriores de Francia. Además, el estudio de los materiales líticos realizados en 2007 fue apoyado por el proyecto ECOSCONICYT C04H01.

Se agradece también al CEQUA - Centro de Estudios del Cuaternario de Fuego Patagonia por la datación de la unidad estratigráfica V (1,45 m, cuadrícula F15).

\section{BIBLIOGRAFÍA}

BERTRAN P. 2009. Estudio geológico preliminar del alero rocoso Cerro Castillo (Patagonia austral, Chile). Magallania, vol. 37(1): 163-172.

BIRD, J. 1993. Viajes y Arqueologia en Chile Austral. Ed. Univ. de Magallanes, Punta Arenas (Chile).

BORRERO, L. 1997. La extinción de la megafauna en la Patagonia. Magallania 25: 89-102.

CHADEFAUX, C. 2006. Altérations physico-chimiques des matériaux archéologiques. Centre de Recherche et de Restauration des Musées de France. Master de Chimie. Univ. Pierre et Marie Curie, 70p. Paris. multigraphié.
CURRY, P. 1991. Distribucion de sitios e implicaciones para la movilidad de los canoeros en el Canal Messier. Anales del Instituto de la Patagonia, Punta Arenas (Chile), vol. 20 : 146-154.

EMPERAIRE, J. y A. LAMING, (s.f) Note de travail, Route de Cerro Castillo à Rio Paine. Archives Emperaire doc. $n^{\circ} 58$, dactilografíado.

GAETE, N., NAVARRO, X., CONSTANTINESCU, F., MERA, C., SELLES, D., SOLARI, M-E., VARGAS, M-L., OLIVA, D. y L. DURAN. 2004. Una mirada al modo de vida canoero del mar interior desde Piedra Azul. Chungara, Revista de Antropologia Chilena, Volumen Especial: 333-346.

GUIDON, N. y LABOUDIE. non daté Fiches typologiques $d u$ site de Rio Condor. Archives Emperaire doc. n³0, dactilografíado.

HADJUK, K, A. y M. LEZCANO. 2005. Un "nuevo-viejo" integrante del elenco de instrumentos óseos de Patagonia: los machacadores óseos. Magallania, Año 2005, vol.33, no.1, p.63-80.

LANGLAIS, M. Y F. MORELLO. 2009. Estudio tecno-económico de la industria lítica de Cerro Castillo (Provincia de Última Esperanza, Chile) Magallania, vol. 37(1): 61-83.

LEGOUPIL, D. y P. BERTRÁN. 2006. L'abri Pedro Cárdenas et l'abri de Cerro Castillo. Mission archéologique de Patagonie: rapport dactyl. 41p.

LEGOUPIL, D. y P. SELLIER. 2004. La sepultura de la Cueva Ayayema (Isla Madre de Dios, archipiélagos occidentales de Patagonia). Magallania, vol. 32, Punta Arenas, Chile : $115-124$.

LEGOUPIL, D., BEAREZ, P., LEPETZ, S., SAN ROMAN, M. y K. SALAS. 2007. De la pesca a la caza: evolución económica del sitio Stuven 1, al sur del Golfo de Penas. En: Arqueología de Fuego-Patagonia. Levantando piedras, desenterrando huesos... y develando arcanos. Editado por F. Morello, M. Martinic, A. Prieto y G. Bahamonde. Ediciones CEQUA Punta Arenas: 295-310.

NAMI, H. 1987. Cueva del Medio : Perspectivas arqueológicas para la Patagonia austral. Anales del Instituto de la Patagonia, Sr. Cs. Soc., Punta Arenas (Chile), vol. 17: 73-106.

PRIETO, A., CANTO, J. y J. PRIETO. 1991. Cazadores tempranos y tardíos en la Cueva 1 del Lago Sofía. Anales del Instituto de la Patagonia, Sr. Cs. Hs, Punta Arenas (Chile), vol. 20 : 75-99.

PRIETO, A. 1994. Arqueria selk'nam. La guerra y la Paz en Tierra del Fuego. Ed. Colegio Pta Arenas., 123p.

PORTER, C. 1993. GUA- 010, Un sitio costero erosionado en una zona sísmica activa. En: Actas del XII Congreso 
Nacional de Arqueología Chilena. Tomo I, Simposios. Boletín del Museo Regional de la Araucanía.

RIVAS, P., OCAMPO, C. y ASPILLAGA, E. 1999. Poblamiento temprano de los canales patagónicos: el núcleo ecotonal septentrional. Anales del Instituto de la Patagonia, Ser. Cs. Soc., Punta Arenas (Chile), vol. 27: 221-230.

SAN ROMAN, M. y F. MORELLO. 2003. Nota sobre una excavación de sondeo en el alero de Cerro Castillo 1 , Provincia de Última Esperanza, Magallanes (Chile). Magallania, vol. 31, p. 139-147.
SCHIDLOWSKY, V. 2001. Les premiers chasseurs maritimes et les chasseurs terrestres de Patagonie australe: Comportements techno-économiques et identité culturelle - Contribution de la technologie lithique. BAR Internat. Ser. 954, Paris Monograph in American Archaeology, 10, 344p.

SOLARI M. E. 2009 Análisis antracológico de una columna estratigráfica del alero cerro Castillo (Magallanes, Chile). Magallania, vol. 37(1):157-162. 